\title{
Pronations-/Abduktionsfraktur (nach Lauge-Hansen) des Malleolus lateralis (AO 44 C1) mit konsekutiver Nonunion und Syndesmoseninsuffizienz
}

\author{
Reto Babst
}

\section{Fallbeschreibung}

54-jähriger übergewichtiger Versicherungskaufmann stolpert anlässlich eines Faustballspiels und macht dabei wahrscheinlich eine Pronations-/Abduktionsbewegung des linken OSG. Bei rasch zunehmender Schwellung wird der Patient 5 Stunden nach Unfall zugewiesen.

Das linke Sprunggelenk präsentiert sich mit einer Subluxation mit ausgeprägter Schwellung und kontusionierter Haut über dem Malleolus medialis. Aufgrund der ausgeprägten Schwellung und der Subluxationsstellung wurde die Indikation zur temporären Stabilisierung mit einem gelenküberbrückenden Fixateur externe gestellt (Abb.1).

Nach abschwellenden Maßnahmen mit Lymphdrainageverband wurde die operative Stabilisierung dieser AO-44C1-Fraktur 5 Tage später vorgenommen. Medialseits fand sich eine trockene Kruste, ansonsten waren die Weichteile gut abgeschwollen. Die Osteosynthese erfolgte mit einer lateralen 1/3-Rohr8-Loch-Platte, ergänzt mit einer a.-p. 2,7-mm-Zugschraube. Eine anatomische Rekonstruktion der Zwischenfragmente gelang nicht. Die Länge und Rotation der Fibula wurden jedoch hergestellt. Die vordere Syndesmose wurde mit 3 Adaptationsnähten genäht. Der Stresstest der Syndesmose mit dem Einzinkerhaken ergab keine Instabilität der Syndesmose, weshalb auf eine Stellschraube verzichtet wurde. Laut Operationsbericht war die Malleolengabel intraoperativ symmetrisch. Die postoperative Behandlung erfolgte in fixierter Orthese und $15 \mathrm{~kg}$ Teilbelastung während 6 Wochen.

OP-JOURNAL 2008; 24: 258-259

(c) Georg Thieme Verlag KG Stuttgart • New York DOI 10.1055/s-2008-1039071
Bereits die erste postoperative Kontrollaufnahme zeigte eine etwas erweiterte mediale Malleolengabel (Abb.2). Aufgrund der intraoperativ stabilen Syndesmosenverhältnisse wurde aber auf eine Revision verzichtet. Bei gleichbleibenden Verhältnissen nach 6 Wochen wurde die Belastung bis zur Vollbelastung nach 8 Wochen erlaubt.

Anlässlich der 3-Monats-Kontrolle ging der Patient stockfrei und hatte eine Gehstrecke von ca. $1 \mathrm{~km}$ bei voller Arbeitsfähigkeit für Bürotätigkeit. Klinisch zeigte sich jedoch ein deutlich geschwollenes OSG mit reizlosen Narbenverhältnissen. Die Beweglichkeit war mit einer Dorsalextension/Plantarflexion von $20 / 0 / 55^{\circ}$ gut. Im Röntgenbild zeigte sich eine Zunahme der Gelenkgabelasymmetrie mit gleichzeitiger Nonunion der Fibula (Abb. 3).

Die Nonunion wurde als Folge der lokalen Devaskularisation der Fragmente und der ungenügenden Stabilität der Syndesmose interpretiert. Deshalb wurde dem Patienten die Reoperation empfohlen. 3,5 Monate nach der Fibulaosteosynthese wurde zuerst das Deltaband medial revidiert. Letzteres war vernarbt. Lokal wurden Verklebungen gelöst und Narbengewebe aus dem medialen Gelenkspalt entfernt. Nach der Osteosynthese-Materialentfernung werden die Frakturränder angefrischt und mit Spongiosa aus der medialen Tibiametaphyse augmentiert. Danach wurde eine 3,5-mm-LCP aufgebracht und die Nonunion durch eine exzentrisch eingebrachte Kortikalisschraube unter Kompression gebracht. Die Fibula wurde mit einer Weber-Zange in der Inzisura fibularis positioniert und bei symmetrischen Gelenkverhältnissen mit einer Stellschraube gehalten (Abb.4).

Drei Wochen nach Intervention Evakuation eines infizierten Hämatoms. Nach Revision mit Jet-Lavage und Antibio-

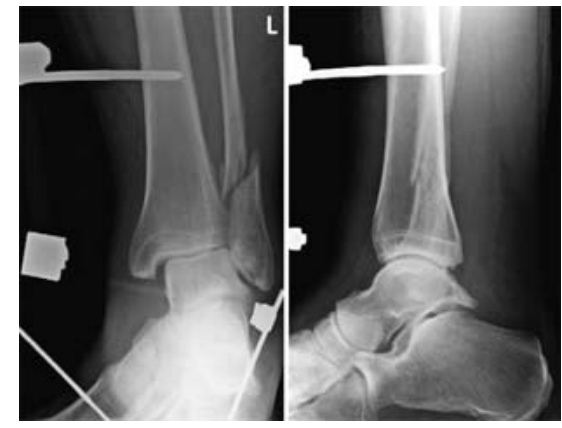

Abb. 1 Status nach Anlage des gelenküberbrückenden Fixateur externe.

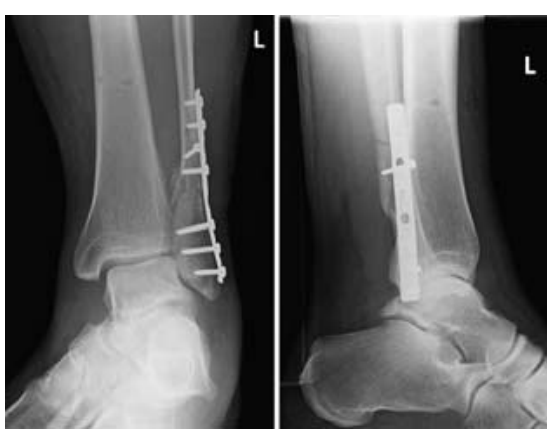

Abb. 2 Röntgenkontrolle unmittelbar postoperativ. Das Gelenk erscheint asymmetrisch. Bei intraoperativ stabiler Syndesmose wird der Befund nicht revidiert.

tikatherapie während 12 Wochen folgenlose Abheilung. Die Stellschraube wurde 12 Wochen nach Reintervention entfernt (Abb. 5).

Ein Jahr nach der letzten Operation wurde das Osteosynthesematerial entfernt. Der Patient war anlässlich der letzten Kontrolle beschwerdefrei, hatte eine unlimitierte Gehstrecke und trieb wieder Mannschaftssport. Das OSG war stabil und hatte einen Bewegungsumfang für die Dorsalextension/Plantarflexion von $15 / 0 / 55^{\circ}$. 


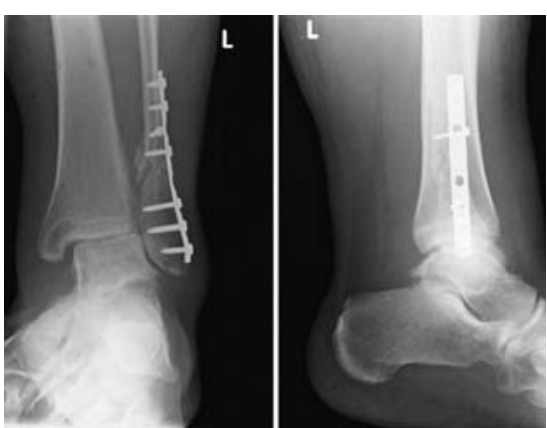

Abb. 3 Röntgenkontrolle 3 Monate postoperativ mit Zunahme der Gelenkasymmetrie und Nonunion der Fibula.

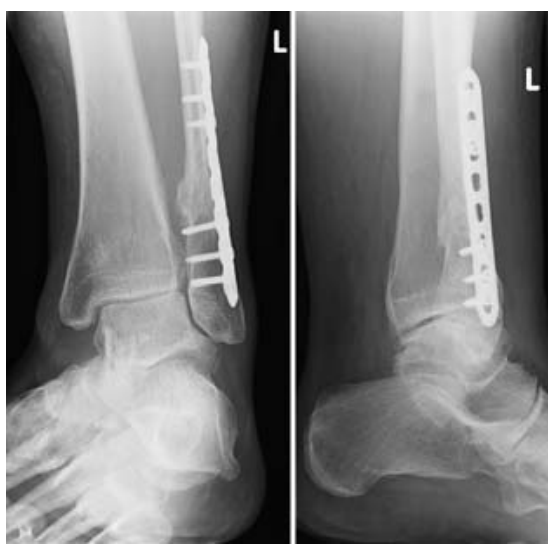

Abb. 5 Röntgenkontrolle 1 Jahr nach Reintervention und Stellschraubenentfernung 12 Wochen nach Revisionsoperation.

\section{Diskussion}

Wie hätte die Komplikation der Nonunion mit Malleoleninsuffizienz vermieden werden können?

Durch eine Revision und Naht des Deltabands?

Eine mediale Revision ist dann indiziert, wenn die Fibula nicht anatomisch rekonstruiert werden kann und eine Ge-

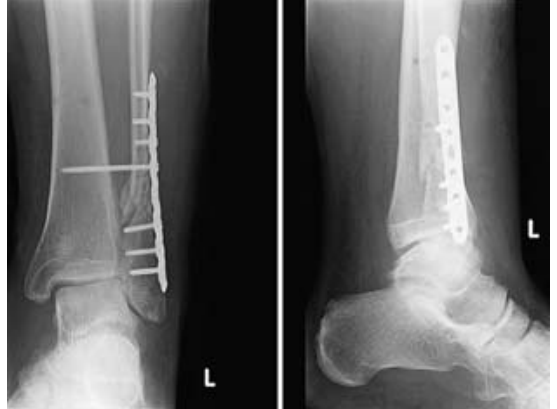

Abb. 4 Röntgenkontrolle nach Revisionsoperation mit Spongiosaplastik, 3,5-mm-LCP und Stellschraube.

lenkasymmetrie besteht [1]. Ein medial erweiterter Gelenkspalt im Bereich des Malleolus medialis ist jedoch kein sicherer Hinweis für eine Ruptur des Deltabands, dies zeigen auch arthroskopische Untersuchungen bei Pronations-Eversionsfrakturtyp [2]. Zudem konnte Maynou in einer zwar retrospektiven Studie zeigen, dass weder ein subjektiver noch ein objektiver Unterschied besteht zwischen der Patientengruppe mit oder ohne Naht des Lig. deltoideum [3] nach einer entsprechenden Fraktur.

Durch die Stabilisierung mit einer Stellschraube?

Malleolarfrakturen vom Pronations-/ Abduktionstyp nach Lauge-Hansen haben die höchste Rate an Nonunionen der indirekten Frakturtypen der Malleoli [4]. Zur Vermeidung lokaler Devaskularisation der Trümmerzone schlagen diese Autoren eine Brückenplatten-Osteosynthese vor. Mit diesem Vorgehen kam es in allen 12 berichteten Fällen, davon erhielten 9 eine Stellschraube, zur folgenlosen Frakturabheilung ohne Hinweise für eine insuffiziente Stabilität der Malleolengabel.
Fazit

Patienten mit einer lateralen Malleolarfraktur AO 44 C1 vom Pronations-/Abduktionstyp nach Lauge-Hansen würde ich heute, unabhängig vom intraoperativen Befund beim Stresstest, primär mit einer Brückenplattenosteosynthese (z.B. mit einer 3,5-mm-LCP) und die Syndesmosennaht mit einer Stellschraube sichern. Eine mediale Revision würde ich nicht primär vornehmen, wenn eine adäquate Reposition der Fibula erreicht wird.

\section{Literatur}

${ }^{1}$ Hahn DM, Colton C. Malleolar fractures. 6.4 Medial exploration. In Rüedi E, Buckley T, Moran RE, eds. AO principles of fracture management. 2nd ed. Stuttgart, New York: Thieme Verlag; 2007

2 Schuberth JM, Collman DR, Rush SM, Ford LA. Deltioid ligament integritiy in lateral malleolar fractures: a comparative analysis of arthrosopic and radiographic assessments. J Foot Ankle Surg, 2004; 43: 20-29

3 Maynou C, Lesage P, Mestdagh H, Butruille Y. Faut-il traiter les lésions du ligament latéral interne dans les équivalents de fracture bimalleolaire? Rev. Orthop Reparatrice Appar Mot 1997; 83: 652-657

${ }^{4}$ Siegel J, Tornetta 3rd P. Extraperiosteal plating of pronation-abduction ankle fractures. JBJS Am 2007; 89: 276-281

Prof. Dr. med. Reto Babst

Chefarzt Unfallchirurgie

Department Chirurgie

Kantonsspital Luzern

6002 Luzern

Schweiz

E-Mail: reto.babst@ksl.ch 